\title{
Modern Methodologies to Assess the Olive Oil Quality
}

\author{
Giovanni Sindona and Domenico Taverna
}

Additional information is available at the end of the chapter

http://dx.doi.org/10.5772/52142

\section{Introduction}

\subsection{Food quality and safety assessment}

Food quality and safety assessments are the main issues that modern food industries are required to fulfill in their production chains, being these properties directly correlated to the claims of a holistic nutrition representing the fundamentals of a healthy balanced diet. In the same direction goes, very recently, the need of checking the origin of an aliment even this is aspect is apparently not directly correlated to the main issues above presented.

All the steps of olive production chain require, as for any other food, a careful evaluation of the identity and absolute amounts of micro and macro components at the "molecular level".

Protect Denomination of Origin (P.D.O.), Protected Geographical Identification (I.G.P.) and other acronyms associated to olive oil quality, are often based on the apparent evaluation of macroscopic properties which hardly deals with a scientific approach to the problem, and whose official protocols of analysis lack often of the required specificity. A case study might be represented by the food safety incident worldwide known as the melamine milk scandal. Hundreds of babies fed with milk diluted with water and fraudulently treated with melamine to artificially increase the apparent protein content, developed kidney stones. Melamine is a plasticizer of molecular formula $\mathrm{C}_{3} \mathrm{H}_{6} \mathrm{~N}_{6}$ thus with a nitrogen content equal to $67 \%$ of its molecular weight. The international official protocols for protein assay are still based on the two hundred years old Kjeldahl method, whereby the nitrogen content is evaluated from the amount of the released ammonia. This was the fate of melamine too! Exactly in the same period researchers from the University of Shangai have published a mass spectrometric assay of melamine in milk based on the specificity of isotope dilution method by means of a contemporary, reliable and affordable approach. ${ }^{1}$

The assessment of quality and safety of all the steps of a given food chain needs to be based on the exploitation of high-tech analytical methodologies. Mass spectrometry represents a 
valuable tool widely applicable to the identification and assay of those quality markers present in olive oil.

\subsection{The principle of mass spectrometry}

Mass spectrometry (MS) is an analytical technique based on the principle of gas phase ion chemistry which allows the identification of analytes of any molecular weight and in any physical state. The availability of (i) multifaceted sample preparation, (ii) high throughput methods for on-line multistep mixture separation, (iii) multistep (MS/MS) ion analysis, (iv) high resolution acquisition and (v) specific isotopic analysis, confer to MS the unique feature of a methodology that can be directly applied to complex natural mixtures such as olive oil.

\subsubsection{Multifaceted sample preparation}

Any sample preparation protocol for MS evaluation of specific properties of foods requires versatile, reliable and affordable approaches which suit structure properties and physical state of the examined analytes. The sample preparation methods may include extensive chemical modification, solvent extraction and different concentration approaches as in the case of triglyceride (TGA) analysis from the alkyl esters of the corresponding fatty acid constituents. This approach classically transform low vapor pressure species to relatively volatile molecules amenable to gas-chromatographic (GC) separation followed by electron impact (EI) ionization and single quadrupole MS analysis. It should be mentioned, even this aspect goes beyond the scope of the present review, that the interest in the direct transformation of TGAs into alkyl fatty ester mixtures, including the methyl derivative (FAME) has found quite recently an enormous interest in the obtainment of biofuels under chemical and/or enzymatic catalysis. ${ }^{2}$<smiles>[R]c1cc(CCOC(=O)CC(C(=CC)C([R])C=O)C(C=O)C([R])C=O)ccc1CCOC(=O)CC1C(C(=O)OC)=COC(OC2OC(CO)C(O)C(O)C(O)C2O)C1=CC</smiles>

3,4

Scheme 1. Deglycosylation of secoiridoids 1,2 leading to the dealdehydes 3, 4 . 
When minor compounds or active principles present in olive oil need to be identified the sample preparation usually requires simple extraction of the analytes followed usually by a direct injection into a multistep mass spectrometer (MS/MS). A case study is represented by the anti-inflammatory dialdehydes formed in olive oil by enzymatic degradation of secoiridoids (scheme 1$){ }^{3}$

A simple check on the existence of these molecules in olive oil can be performed from the crude methanol extracts by means of LC-MS/ MS (Figure 1). ${ }^{4}$

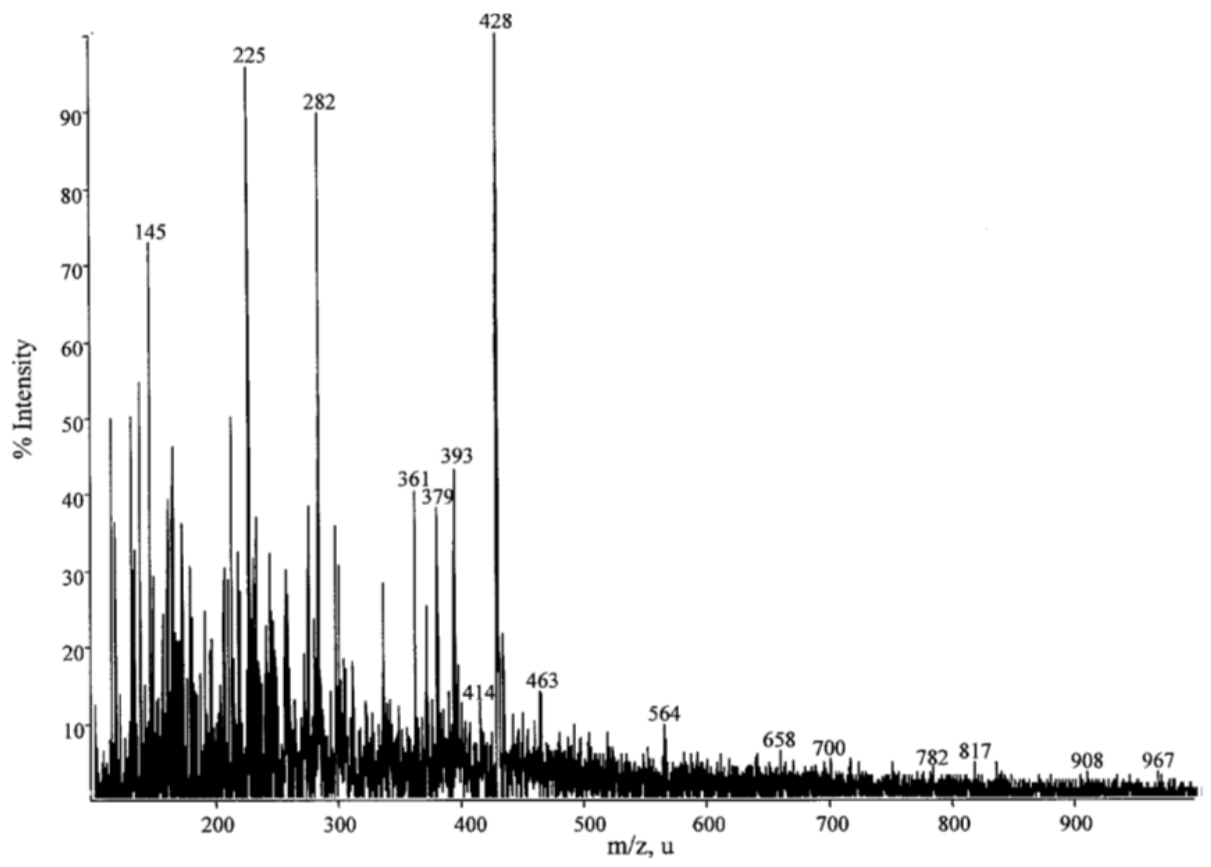

Figure 1. LC-MS chromatogram of a methanol extract.

The structure of many of the analytes present in the figure 1 was evaluated by further MS/MS experiments. The species at $\mathrm{m} / \mathrm{z} 379,414$ and 428 were, than, associated to the protonated dialdehyde 4 ( $\mathrm{R}=$ hydroxytyrosol, Scheme 1$)$ and to the ammoniated species of the corresponding geminal diol and hemiacetal. The latter are expected reaction products of the very reactive system represented by the dialdehydes 3 and 4 , when exposed to water and methanol interaction. The observed results could be considered as a remarkable case of freezing fast equilibrating species and detect them on-line, separately; the formation, however, of stable derivatives from a given analyte, present in a complex mixture such as olive oil, represents a false positive which can be eliminated by selecting a proper derivatization procedure as it will considered later. ${ }^{5}$

The recently introduced MS ambient ionization methods ${ }^{6}$ are applicable to direct evaluation of complex food matrices such as olive oil. In this case too, especially in the assay of a specific component, in situ derivatization may still be needed. ${ }^{7}$ 
When the isotope dilution approach is considered either for quantitative investigation or for a reliable identification of analytes in complex natural mixtures ${ }^{8}$, sample preparation requires the addition of suitable labelled internal standards which should not undergo intramolecular or intermolecular isotope scrambling.

\subsubsection{High throughput methods for on-line multistep mixture separation}

The exploitation of MS coupling with separation techniques was initially hampered by the experimental parameters of a conventional ion source operated at high vacuum. The availability of multistage mass spectrometers has favored direct injections protocols of even complex mixtures, nevertheless, many applications require the on-line separation of each analyte before being submitted to identification and assay. Modern protocols are based on the combination of GC, HPLC, capillary electrophoresis, and other separation techniques directly linked MS/MS instruments. Ionization techniques such as electrospray (ESI), atmospheric pressure chemical ionization (APCI), matrix-assisted laser desorption ionization (MALDI), and differently performing ambient ionization devices are nowadays mainly used in food analysis. The role of GC, as well as HPLC in high-resolution separation of complex mixtures is unquestionable. Similarly, mass spectrometry has attained an indisputable position in analytical chemistry as a highly structure-specific technique that can provide structural identity of a wide range of compounds. Both chromatography and mass spectrometry have, however, their limitations as stand-alone operations. The separation power of any chromatography system is, actually, finite since it will be nearly impossible to achieve complete separation of all components of a complex mixture. The identification of a compound from a chromatographic peak is less than reliable when it has to be indentified by comparing its retention time with that of a standard reference. Therefore, the commonly used separation techniques such as GC and LC cannot provide unequivocal identity of the analyte when used with conventional detection systems. A compound-specific detector is thus an essential adjunct to characterize unambiguously the components that elute from any separation system. In this respect, mass spectrometry offers the unique advantages of high molecular specificity, detection sensitivity, and dynamic range. Mass spectrometry, only, has the advantage to provide confirmatory evidence of an analyte because of its ability to distinguish closely related compounds on the basis of the molecular mass and structure-specific fragment ion information formed by gas phase processes. The confidence in identification of a target compound, however, diminishes when it is present in a mixture, such as olive oil. Because of the universal nature of mass spectrometry detection, the data obtained might also contain signal due to other components of the mixture. The coupling of a separation device with mass spectrometry thus benefits mutually. The result is a powerful two-dimensional analysis approach, where the high-resolution separation and the highly sensitive and structure-specific detection are both realized simultaneously. Some of the benefits that accrue when a separation technique and mass spectrometry are coupled can be summarized as follows:

- The capabilities of the techniques are enhanced synergistically; both instruments may, therefore be operated at subpar performance levels without compromising the data outcome. 
- The high selectivity of mass spectrometry detection allows one to identify co-eluting components.

- The certainty of identification is enhanced further because, in addition to the structurespecific mass spectral data, the chromatographic retention time is also known.

- Multicomponent samples can be analyzed directly without prior laborious off-line separation steps, resulting in a minimal sample loss and saving of time.

- The sensitivity of analysis is improved because the sample enters the mass spectrometer in the form of a narrow focused band.

- Less sample is required than the amount required for off-line analysis by the two techniques separately.

- Because of the removal of interferences, the quality of mass spectral data is improved and any mutual signal suppression is minimized.

Various chromatographic techniques have been employed for separation, preparative isolation, purification and identification of such kind of compounds in food. At date, comprehensive two-dimensional separations are a definite trend in chromatography. Comprehensive two-dimensional gas chromatography (GC $\times \mathrm{GC})$ emerged some 15 years ago. ${ }^{9}$ The latter technique allows for the separation of complex mixtures of volatile analytes GCxGC delivers more GC information in a shorter time than do other methods. GCxGC, at its roots, is a multidimensional separation technique in which the resolving power of two or more different columns is applied to some or all of the components in a sample. As the name implies, comprehensive separations apply all the available resolving power of both columns to all the peaks in a sample. In GCxGC each peak transits the first column, is trapped at the end of the first or the beginning of the second column and is released onto the second column (Figure 2).

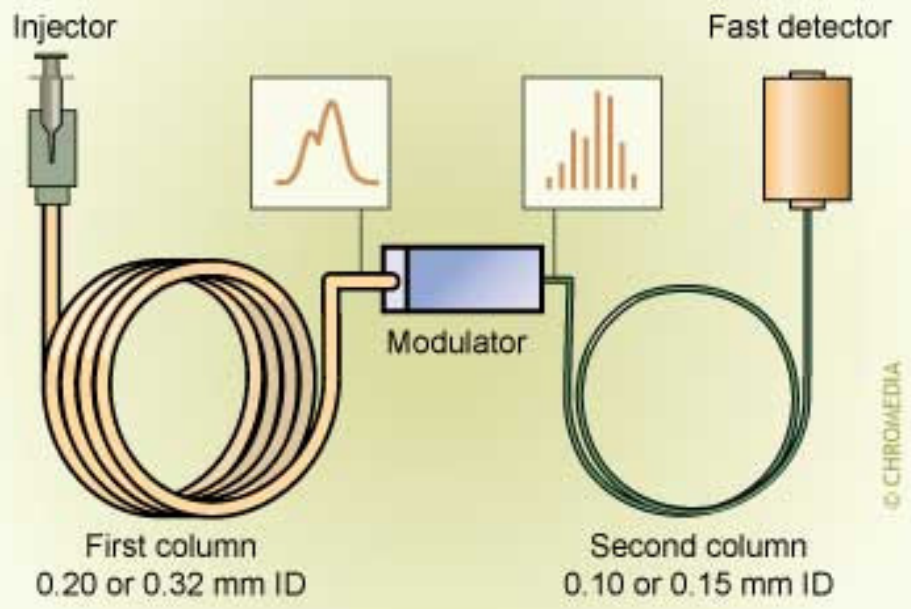

Figure 2. GCxGC device. 
The major difference between GCxGC and heartcutting is that for GCxGC the trapping time and second column separation speeds are fast enough so that many heartcuts can be analysed in the second column during the course of a single first-column run. The heartcuts can be taken rapidly enough that individual peaks from the first column are sliced across several sequential secondary column runs, but going much faster than that has no advantage. Achieving this level of performance requires a high-speed separation for the secondary column and encourages ordinary speeds for the first column. Faster separations on the first column can drive the second-column speed requirement higher than what is practical. So, the main advantages of the technique are vastly-expanded separation space and the ability to resolve hundreds or thousands of peaks; improved sensitivity; and more obvious structure in retention patterns for peaks. The increase in sensitivity is due to the fact that most modulators compress and focus peaks into narrow bands, improving the signal, and at the same time separate coeluting materials from the analyte of interest, decreasing the noise.${ }^{10}$ Comprehensive Two-dimensional Gas Chromatography (GCxGC).${ }^{11}$ Comprehensive two-dimensional separation is achieved also by liquid chromatography; the roots of comprehensive two-dimensional liquid chromatography (LC $\times$ LC) are older. Twodimensional thin-layer chromatography can be considered a form of $\mathrm{LC} \times \mathrm{LC}$, dating the technique back at least some 50 years. The contemporary version of LC $\times \mathrm{LC}$ was first demonstrated by Erni and Frei. ${ }^{12}$ The common way to perform LC $\times$ LC involves a firstdimension separation that is relatively slow, with a typical analysis time of $1 \mathrm{~h}$ or longer. Fractions of the effluent from this first-dimension column are collected in a loop of a switching valve. When this valve is switched, the fraction is injected onto a second column, which provides a much faster separation, typically with an analysis time of a minute or less.



Dimension 2
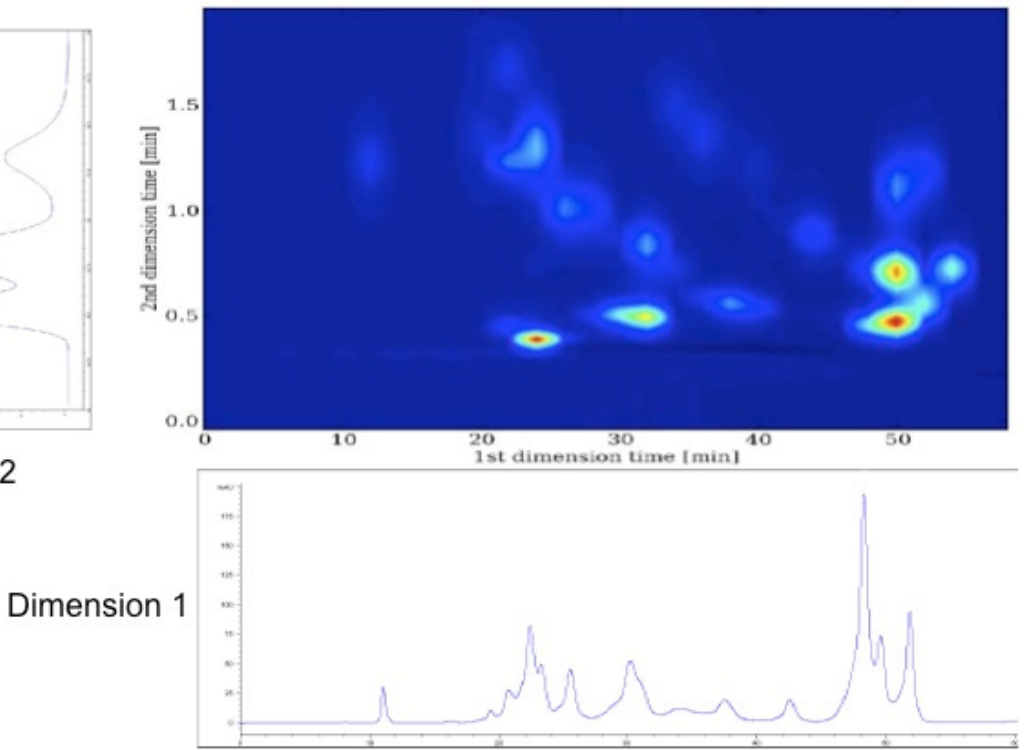

Figure 3. LCxLC, 2 dimensions chromatograms. 
While one fraction is being analyzed on the second-dimension column, a new fraction is being collected in another loop connected to the same valve. The result of a comprehensive two-dimensional separation is a series of many (for example, 100) fast second-dimension chromatograms (Figure 3).

\subsubsection{Multistep (MS/MS) ion analysis}

The concept of in-space tandem mass spectrometry is illustrated in Figure 4. It involves two mass spectrometry devices; the first one (MS-1) performs the mass selection of a desired target ion from a stream of ions produced in the ion source. This mass-selected ion undergoes either unimolecular fragmentation (equation 1) or a chemical reaction in the intermediate region.

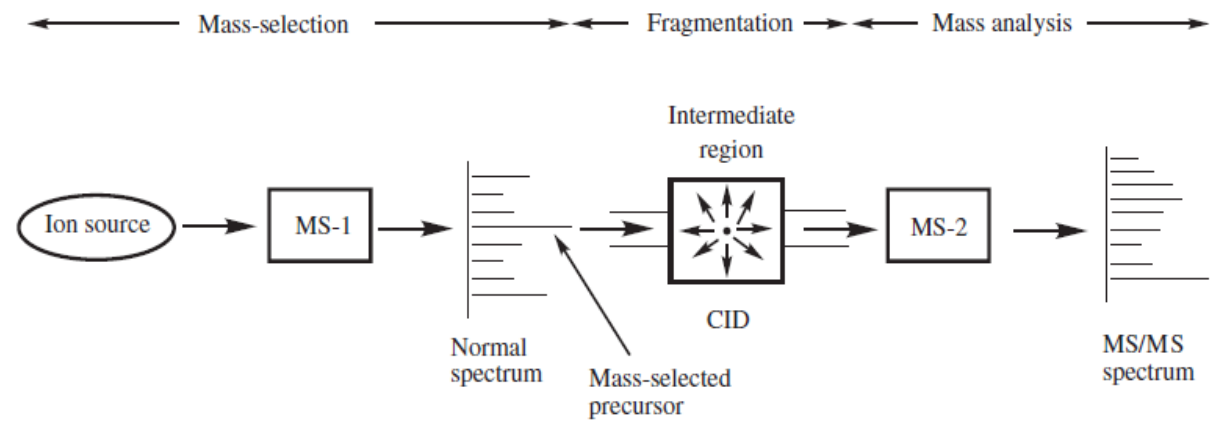

Figure 4. Basic principle of tandem mass spectrometry.

The second MS system (MS-2) performs the mass analysis of the product ions that are formed in the intermediate step.

$$
\begin{aligned}
m_{p}{ }^{+} & \longrightarrow m_{1}^{+}+m_{n}^{\prime} \\
& m_{2}{ }^{+}+m_{n}^{\prime \prime} \\
\longrightarrow & m_{3}{ }^{+}+m_{n}^{\prime \prime \prime}
\end{aligned}
$$

The first stage of MS/MS separates a mixture of ions into individual components thus resolving a mixture of compounds, whereas the second stage acts as an identification system for the mass-resolved ions. By convention, the mass-selected ion is called the precursor ion $\left(m_{p}{ }^{+}\right)$ and its fragments are called product ions (e.g., $\mathrm{mi}_{1}^{+}, \mathrm{m2}^{+}, \mathrm{m3}^{+}$, etc. in equation 1 ). The $m n$ species in equation 1 are neutral losses. Because of the incontrovertible link between the precursor ion and all of its product ions, the molecular specificity of MS/MS is garanteed. This unique attribute of tandem mass spectrometry is a highly useful feature that plays a role in the unequivocal identification of a target compound in real-world samples. Tandem mass spectrometry is not restricted to two stages of mass analysis (i.e., MS/MS or MS²); it is also possible to perform multistage MS (i.e., higherorder MS) experiments, abbreviated as $\mathrm{MS}^{n}$. These experiments can determine the genealogical relation between a precursor and its ionic 
products. For example, $\mathrm{MS}^{3}$ indicates three stages of tandem mass spectrometry, which involves mass selection of one of the products, say either $m_{1}{ }^{+}, m_{2}{ }^{+}$, or $m_{3}{ }^{+}$, formed from the precursor $\mathrm{mp}_{p}^{+}$of the first-stage MS (equation 1), and determination of the second-generation products of that mass-selected ion. Multistage MS experiments are performed mostly with iontrapping instruments. A maximum of $12 \mathrm{MS} / \mathrm{MS}$ experiments has been envisioned with a quadrupole ion trap. Beam-type instruments can also be used for $\mathrm{MS}^{n}$ experiments but require as many discrete mass analyzers as there are number of stages in the experiment, making it difficult to perform more than four stages of MS/MS experiments. As an example, a threesector magnetic field instrument can perform up to $\mathrm{MS}^{3}$ experiments. Practical applications of tandem mass spectrometry require data to be acquired in the following four scan modes. A pictorial representation of these scans and their symbolism is given in Figure 5.

MS-1

Intermediate
region MS-2 Symbolism

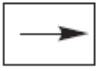

Set at precursor $m / z=m_{\mathrm{p}}$

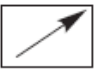

Scanned



Set at $m / z=m_{\mathrm{p}}$ Scanned



Set at precursor $m / z=m_{\mathrm{p}}$

Product-ion scan



$\mathrm{AD}$

Precursor-ion scan

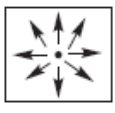

$\mathrm{AD}$

Neutral-loss scan

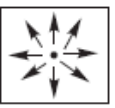

$\mathrm{AD}$

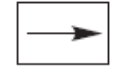

Set at product $m / z=m_{1}$
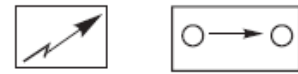

Set at $m / z=m_{\mathrm{p}}-m$ Scanned

Selected-reaction monitoring

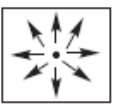

$\mathrm{AD}$
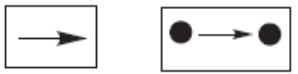

Set at product $m / z=m_{1}$

Figure 5. Representation of four scan modes of tandem mass spectrometry. AD refers to ion activation and dissociation, and the filled and open circles stand for fixed and scanning mass analyzers, respectively.

The product-ion scan (the old, now-unaccepted term, still used by some, is daughter-ion scan) is the most common mode of MS/MS operation. That spectrum is useful in the structure elucidation of a specified analyte. Information obtained in this scan is similar to that derived from a normal mass spectrum, except that the spectrum contains only those product ions 
that are formed exclusively from a mass-selected precursor ion. To acquire this spectrum, the first mass analyzer is set to transmit only the precursor ion chosen, and the second mass spectrometer is scanned over a required $\mathrm{m} / \mathrm{z}$ range. Another popular MS/MS scan is the precursor-ion scan (the past, now-unaccepted term is parent-ion scan). It provides a spectrum of all precursor ions that might fragment to a common, diagnostic product ion. The spectrum is obtained by adjusting the second mass spectrometer to transmit a chosen product ion (e.g., $m 1$ ) and scanning the first mass analyzer over a certain $m / z$ range to transmit only those precursor ions that fragment to yield the chosen product ion. This scan is useful for the identification of a closely related class of compounds in a mixture. A typical example is the detection of Sudan azo dyes within a food. A major product of fragmentation of azo dyes is the ion at $m / z 156$. The precursor-ion scan of this $m / z$ value will detect the presence of azo dyes selectively in a complex mixture (see Figure 6).

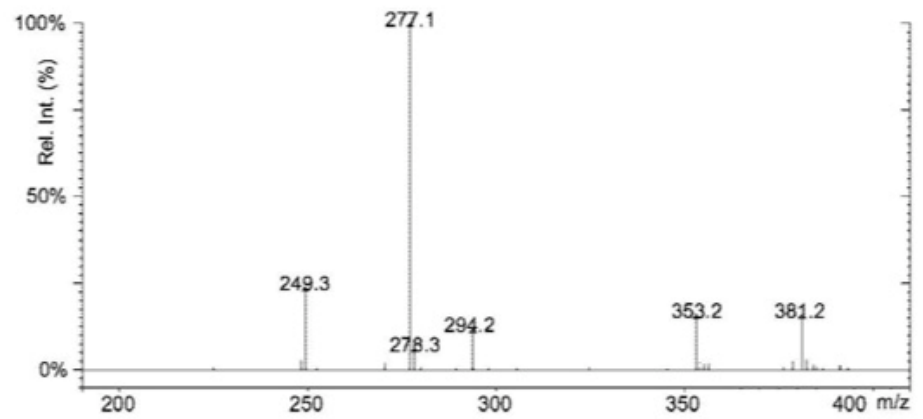

Figure 6. Parent ion scan MS/MS spectrum of the fragment m/z 156 present in the APCI spectra of 5 azodyes extracted from baked products and sampled by flow injection.

In a constant-neutral-loss scan, all precursors that undergo the loss of a specified common neutral are monitored. To obtain this information, both mass analyzers are scanned simultaneously, but with a mass offset that correlates with the mass of the specified neutral. Similar to the precursor ion scan, this technique is also useful in the selective identification of closely related class of compounds in a mixture. For example, the loss of $44 \mathrm{Da}$ is a common reaction of carboxylic acids. Through the constant neutral loss scan, the identity of all carboxylic acids present in a complex mixture can be revealed. Similarly, by monitoring the 98-Da neutral loss, the presence of phosphopeptides can be detected in a complex mixture. ${ }^{13}$ The fourth scan, selected-reaction monitoring (SRM), is useful in quantitative measurements of analytes present in complex mixtures. Conceptually, this scan mode is similar to the product-ionscan. However, instead of scanning the second mass spectrometer in abroad mass range, the two mass analyzers are adjusted to monitor one or more chosen precursor-product pairs of the analyte. This operation is identical to the selected-ion monitoring mode (SIM) of data acquisition. Monitoring more than one reaction is termed multiple-reaction monitoring (MRM). All four scan modes can be implemented with magnetic sector- and quadrupole-based true or hybrid tandem instruments. Time-of-flight (TOF) and tandem-in time devices are also suitable for product scan experiments, but they are unable 
to perform the other three scans. Further, within the multistep tandem mass spectrometry analyses have to be considered also Ion Mobility (IM). Those mass spectrometers are hybrid instruments that combine an IM separation system with conventional MS systems. An ion mobility spectrometer (IMS) can also serve as a stand-alone ion detection system. ${ }^{14}$ A IMS uses gas-phase mobility rather than the $m / q$ ratio as a criterion to separate ions. ${ }^{15}, 16$ The mobility of ions is measured under the influence of an electrical field gradient and cross flow of a buffer gas, and depends on ion's collision cross section and net charge. A typical IMS consists of a reaction region and a much longer drift region; both regions contain a series of uniformly spaced electrodes that are connected via a series of high resistors to provide a uniform electric field strength. The two regions are separated by an electrical shutter. Buffer gas is also circulated in the drift tube. The ions are generated in the reaction chamber and are allowed to enter the drift region by opening the electrical shutter for a brief period. Under the influence of an electrical field, ions drift into the drift tube, where they are separated according to their size-to-charge ratio. The mobility of ions is a combined effect of ion acceleration by the electric field and retardation by collisions with the buffer gas. At the end of the drift region is placed an ion detector (e.g., a Faraday cup) for the detection of the separated ions. For more accurate mass analysis, an IMS is coupled to a quadrupole or TOF mass analyzer. ${ }^{17,18}$

Similar to LC-MS systems, the IMS serves as a separation device and the quadrupole or TOF mass analyzer as a detection device, but has the added advantage that separation times are in milliseconds. ESI and MALDI ion sources have both been coupled to IM-MS instrument. 19, 20 Latter consists of a MALDI source, an ion mobility cell, a CID cell, and a TOF mass analyzer. Ions exit the drift tube when the axial field strength of the ion mobility cell is ramped up, and enter the source region of a-TOF instrument, where the ions are detected intact in the usual manner. Alternatively, ions can be fragmented in the CID prior to their detection by the TOFMS. The resolution of an IMS, usually very low (10 to 12) can be increased to 200 to 400 by increasing the pressure of the buffer gas, connecting the ion source directly with the drift tube, increasing the length of the drift tube, and increasing the electric field gradient of the drift tube. Unlike LC/MS separation, an ion chromatogram can be obtained within $1 \mathrm{~s}$.

\subsubsection{High-Resolution Acquisition}

High-resolution mass spectrometry (HRMS) strongly competes with the classical tandem MS, in particular in the field of quantitative methods (e.g., pesticides, drugs, etc). High-res analyses are commonly used for residues in food: the availability of new and more sensitive, selective instrumentation contributed to the development and the acceptance of such technology in the last few years. Many benefits are gained by using HRMS, including the collection of full-scan spectra, which provides greater insights into the composition of a sample. High-res instruments, including double focusing, FTICR, orbitrap mass spectrometers, are able to measure the mass of an ion and its associated isotope peaks with sufficient accuracy to allow the determination of its elemental composition. This is possible because each element has a slightly different characteristic mass defect. The accurate mass measurement shows the total mass defect and provides a means to determine its elemental 
composition. In the last years, the number of studies reposting HRMS-based approaches for food analysis applications had a significant increase. Even if laboratories still analyze pesticides, drugs and other microcomponents by using LC-MS/MS and other older approaches, HRMS instruments are used in both quantitative and confirmative works. Highres instruments allow not only for high resolution, superior sensitivity, larger dynamic range and improved speed. Generally, a high-resolution analysis provides more comprehensive information, e.g. identifying coeluted and therefore potentially suppressing matrix compounds. Selectivity of detection in complex matrices is only ensured when the HRMS instrument can physically resolve the analyte mass from that of other coeluted isobaric matrix compounds. HRMS permits selectivity in their elemental composition and therefore have a different exact mass. Many reports in the literature described the use of a narrow mass window to improve selectivity. ${ }^{21-23}$ Other studies have addressed the issue of the mass resolution required for trace analysis in food matrices..$^{24}$ Resolutions of 50,000 or more FWHM were suggested for low levels of analytes in complex matrices such as animal feed. The same authors concluded that a resolving power of 25,000 FWHM is sufficient for less complex matrices. Their conclusion was based on experiments that involved analyzing matrix samples spiked at low levels with various veterinary drugs, mycotoxins, and pesticides. Moreover, the latest generation of Orbitrap instrumentation provides resolutions well above 100,000 FWHM (Figure 7). Such instrument provides a clearly higher selectivity than QqQ instrumentation.
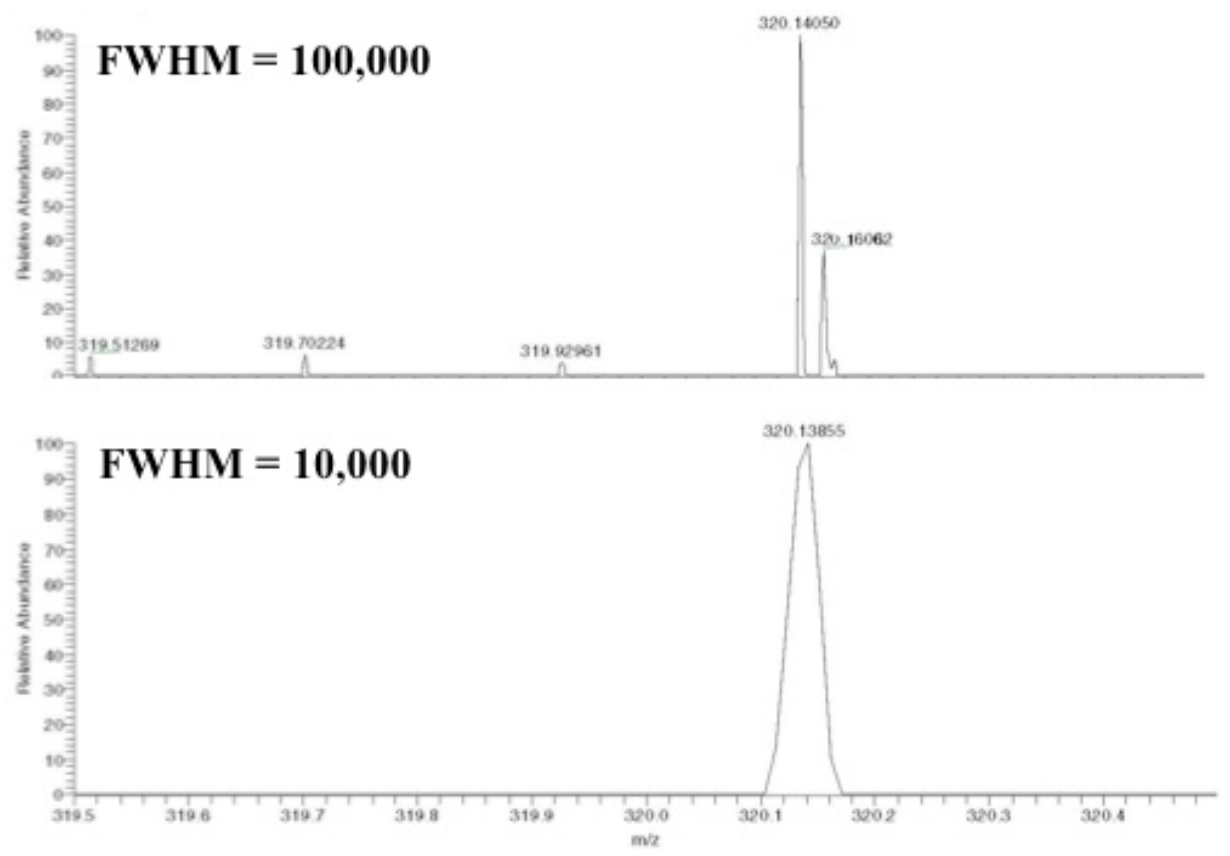

Figure 7. High resolution MS spectra registered by a Orbitrap instrument; the signal at $\mathrm{m} / \mathrm{z} 320$ has been resolved at 100,000 FWHM (top) and at 10,000 FWHM (bottom). The higher resolution allows for a clear separation of the analyte ion from an isobaric matrix compound. The lower resolution does not resolve these two ions. 
The emergence of higher and higher resolving HRMS instruments is a significant advantage over QqQ technology. It is a fact that in the recent past every QqQ instrument generation showed an impressive increase in detector sensitivity as compared with the previous generation. This provided not only lower limits of detection, but also permitted the dilution of extracts in order to reduce signal suppression effects. The other side of the coin is the fact that detection selectivity remained virtually unchanged. This resulted in SRM traces which show many peaks corresponding to compounds being eluted before, after, or even together with the targeted analyte. ${ }^{25}$ Further, most of the currently available literature for HRMS multiresidue screening is based on measurements made with instruments capable of providing resolutions between 10,000 and 20,000 FWHM. ${ }^{26,}{ }^{27}$ Basically, higher resolution leads to superior results when detecting low concentrations of analytes in complex matrices. However, depending on the technology used: TOF instruments, for instance, achieve higher resolution by employing very long flight tubes. This might be a physical limitation. Orbitraps provide a user-defined resolution. There is no relevant sensitivity drop when choosing a higher resolution. However, the resolution obtained is proportional to the measurement time. The resulting consequences are fewer data points per time unit. This is a limitation when using UPLC equipment or when attempting polarity-switching experiments. Furthermore, higher resolutions produce more data which have to be processed by the instrument and later accessed from the hard disk when extracting mass traces from the raw data.

\subsection{Identification and assay of major components of olive oil}

Triacylglycerols (TAG) are the most important lipid component of human diet and represent by far the main constituent of olive oil (96-99\%), they differ in fatty acid composition as well as in their sequence within the glyceride. It is now well established that the biochemistry of lipids assumed with diet, hence those present in olive oil, is strictly related to the action of the digesting enzymes, such a pancreatic lipase, which are able to discriminate between the fatty acids etherifying glycerol at different sites (Figure 8 ). ${ }^{28}$

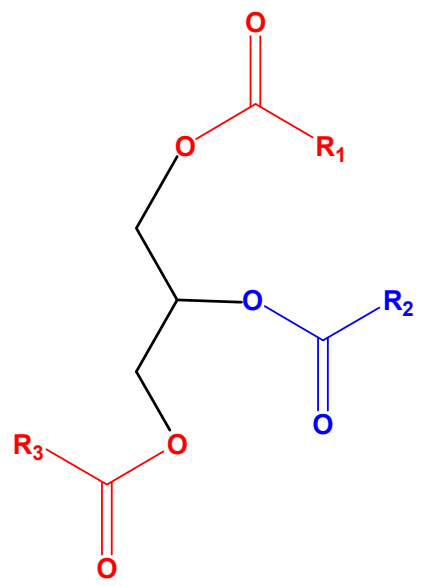

Figure 8. Schematic of TG structure. R, fatty acid, with R1, R2, and R3 indicating the fatty acid at the sn$1,-2$, and -3 positions, respectively. 
Therefore the classic analytical methods based on the identification and assay of methyl or ethyl fatty acid esters obtained by direct transesterification of TGAs, still the official method of analysis, lack by nutritional, hence quality, validation. ${ }^{29}$ Chromatographic ${ }^{30,}{ }^{31}$ and ${ }^{1} \mathrm{H}$ NMR methods ${ }^{32-34}$ have been introduced for a detailed analysis of TGA in olive oils. In a recent application of chromatographic methodologies combined with statistical analysis ${ }^{35}$ an effective discrimination of extra virgin olive oils was achieved by direct injection of the samples without any preliminary chemical derivatization. Atmospheric pressure chemical ionization (APCI), linked to a triple quadrupole, mass spectrometer provided sensitivity to detect over 50 compounds in the sample. In figure 9 is reported the MS profile of a TGA mixture present in a given olive oil sample.



Figure 9. HPLC-MS profile of diluted extra virgin olive oil. The three letter acronyms have the conventional meaning, where O, M. Po, P, S, L, Ln, E. Ma, and Mo, correspond to asingle fatty acid respectively.

A peculiar application to the evaluation of TAG profiling ${ }^{36}$ of Matrix assisted laser desorption (MALDI) time-of-flight (TOF) mass spectrometry was introduced (Figure 10). The method was applied to a peculiar type of olive oil produced in a desert, by means of a conventional 2,5dihydroxybenzoic acid (DHB) matrix, using un-treated samples. The fatty acid composition of the analyzed foodstuff was in agreement with what could be anticipated by applying conventional GC techniques, with the main difference, however, that the presence of oleic acid, for instance, was evaluated through the identification of differently structured TAGs, such as Trioleyl (31.53\%) dioleoyl-palmitoyl (23.06\%) and dioleoyl-linoleoyl (14.31\%) glycerol.

Microbiological studies have recently demonstrated the occurrence in the suspended fraction of freshly produced olive oil of a rich microflora mainly composed of yeasts ${ }^{37}$ which, probably, induces the presence of lipase. ${ }^{38}$ This fact can explain the many changes observed in the triglycerides profiles during aging as a consequence of the lipase action ${ }^{39}$ 


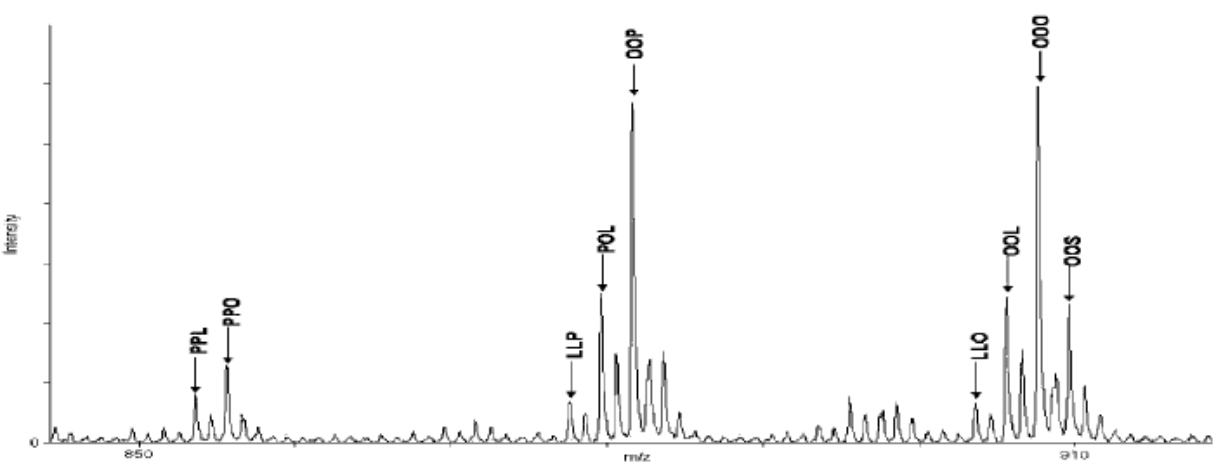

Figure 10. TAG spectrum obtained from MALDI-TOF/MS of an olive oils sample in positive ion mode.

and to the presence of several mono and di carboxylic acids in olive oil derived from many types of biotransformation..$^{40-42}$ The products resulted from this modifications are new type of triglyceride oligopolymers present in olive oil, as it was reported for Japanese wax. ${ }^{43}$

The relative activity of lipase to catalyze hydrolysis and transesterification of triglycerides was studied and confirmed either with model systems mimic olive oil, or directly with the foodstuff. ${ }^{44}$ It was monitored, therefore, by mass spectrometry, the ability of lipase to catalyze the formation of new functionalized triglycerides from triglycerides and free mono and di-acids. The oligomerization of triglycerides has been monitored on real olive oil samples, and verified in solution under lipase catalysis, whose presence in olive oil was already documented. The hydrolytic pathways taken under enzymatic treatment is balanced by the formation of triglycerides oligomers that should not alter the nutritional value of the aliment. As a general view of the lipase action on triglyceride models in the presence of a carboxylic acid, Tripalmitoyl- (1) and trioleoyl (2)- glycerol were incubated with succinic under lipase catalysis at $70^{\circ} \mathrm{C}$ (Scheme 2$)$. The choice of succinic acid was motivated by the known presence of this dienoic acid in olive oil.
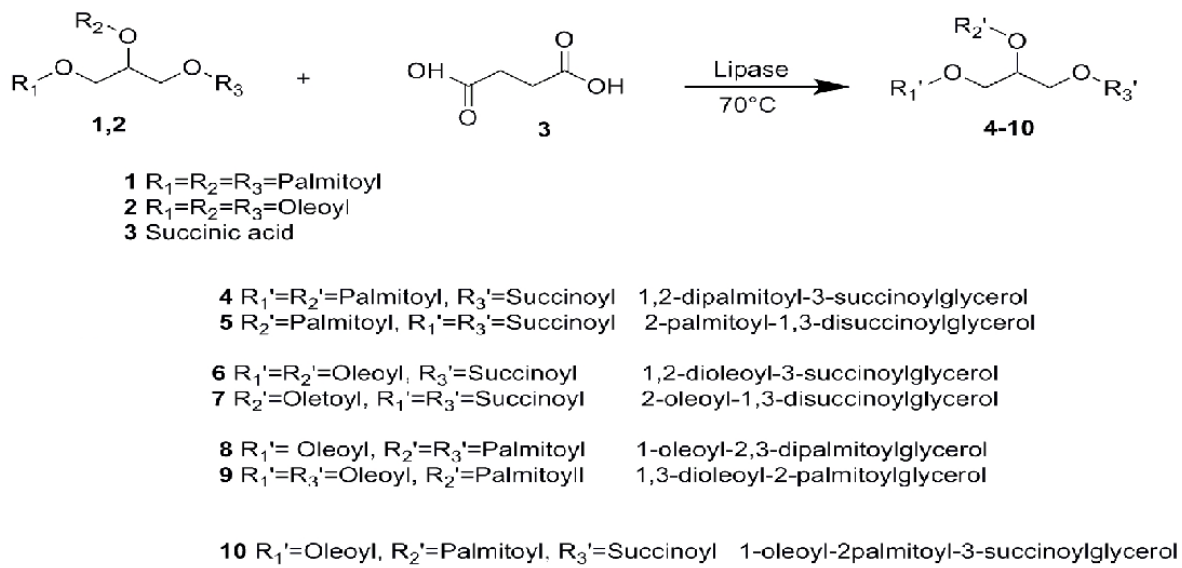

Scheme 2. Incubation of triglyceride mixture with succinic acid in the presence of lipase. 
In Figure 11 is reported the partial full scan spectrum of the succinoyl derivative of of dioleylglycerol observed as sodiated species. The MS peak might correspond to other isomeric species, in the sense that the succinoyl moiety could be linked to different glycerol sites, according to the mechanism of lipase action. Nevertheless, it is clear that after thermal mild treatment, similar to the deodorizing protocol, the presence of lipase and of succinic acid in olive oil can cause, besides TAG isomerization, the formation of side products which could be useful as markers of the industrial procedure.

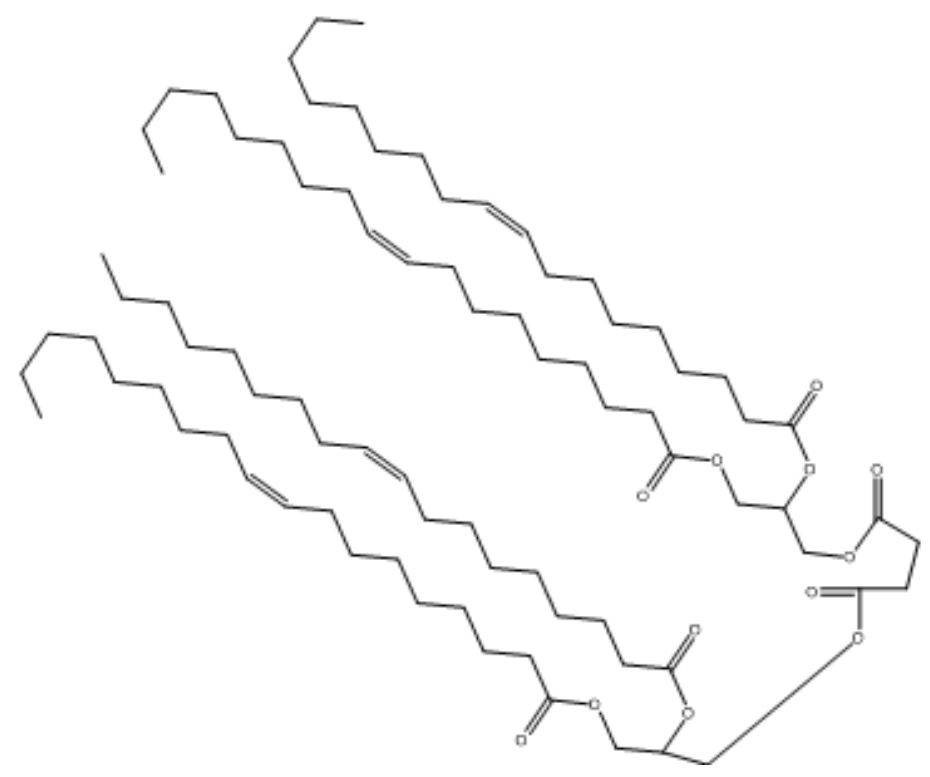

Formula. 1,2-dioleyl-3-succinoylglycerol (6). $\mathrm{C}_{82} \mathrm{H}_{146} \mathrm{O}_{12}$



Figure 11. ESI-MS full scan spectrum of compound 6 identified as $\left[\mathrm{M}+\mathrm{Na}^{+}\right]$adduct. 
The ideal objective of any extraction method is to extract the largest possible amount of matrix constituents without altering their identity. to measure "total fat" various methods have been approved by the regulatory agencies of most major countries. ${ }^{45}$ Most of the older methods involve solvent extraction and gravimetric mass measurement of the lipid residue. Several apparatus have been developed since 1939 for automatic extraction (e.g., Soxhlet, Soxhterm, Soxtec, Butt, etc). ${ }^{46}$ For total fat determination ethyl ether (diethyl ether) is often the solvent of choice as it is relatively non-polar and extracts mostly the non-polar lipids (triacylglycerols, sterols, tocopherols, etc) while poorly extracting the polar lipids (glycolipids and phospholipids). ${ }^{47,48}$ Methods have been developed to use supercritical fluid extraction for total fat determination. ${ }^{49}$

However, because of the diversity of the matrix content, methods are designed to efficiently extract specific molecule classes. The extraction of total lipids in foods is often considered to be a simple procedure; to extract total lipids (non-polar and polar) more polar solvents must be used as extractants (e.g., hexan or petroleum ether, chloroform, methanol, isopropanol, water). The most popular method for total lipid extraction is the Folch method. ${ }^{50}$ Lipids are extracted from a sample by using chloroform-methanol (2:1 by volume). Many modifications to the Folch procedure have been published. ${ }^{51}$

\subsection{Identification and assay of minor component of olive oil}

The quality of olive oil is often associated with the presence of microcomponents whose healing effects have been proved in some special cases. ${ }^{52,}{ }^{53}$ Virgin olive oils contains also phenolic substances responsible for their stability against oxidation. ${ }^{54}$ Phenolic fraction includes simple phenols, tyrosol, and hydroxytyrosol, derivatives of hydroxybenzoic and hydroxycinnamic acids, aglycons of some glucosides, namely oleuropein, demethyloleuropein, ligstroside, and verbascoside. $.5,56$

Absolute analytical methods for mass spectrometric detection as well as for quantification of such bio-active molecules, such as oleopentadialdehydes have been developed. As previously mentioned, the presence of dialdehydes in olive oil can be easily ascertained by a simple LC-MS approach (scheme and figure above). However false positive can be easily formed during the ionization process due to extreme reactivity of the sampled molecules with polar solvents such as water or methanol. ${ }^{57}$ Such novel method provides an in situ chemical derivatization of the whole set of molecules into stable alkyloxime derivates, allowing the concomitant use of a stable isotope standard which improves both the precision and the accuracy of the measurements, thus reducing the drawbacks may arising from the calibration procedure, sample preparation, and matrix effects.

The method was applied to a set of different Italian virgin olive oils showing the presence of 5 and 6 in the range of $70 \div 166$ and $23 \div 132$ ppm, respectively. The same approach was exploited in the assay of hydroxytyrosol (1) and tyrosol (2), the strong antioxidant present in large amount in virgin olive oil, by LC-MS/MS under MRM condition and isotope dilution method, using $\mathrm{d}_{2}$-labelled internal standards, 3 and $\mathbf{4}$, obtained by simple synthetic procedures (Chart 1). This active principles ranged from 10 to 47 and 5 to $25 \mathrm{ppm}$ in experimental and commercial virgin olive oil, respectively. ${ }^{58}$ 
<smiles>[R10]ON=CCC(CC(=O)OCCc1ccc(O)c([R])c1)/C(C=NO[R17])=C/C</smiles>

\section{$5 \mathrm{R}_{1}=\mathrm{CH}_{3}, \mathrm{R}=\mathrm{H} \quad O$-methoxy-pentanedialdoxime-Tyr \\ $6 \mathrm{R}_{1}=\mathrm{CH}_{3}, \mathrm{R}=\mathrm{OH} \quad O$-methoxy-pentanedialdoxime-HTyr $7 \mathrm{R}_{1}=\mathrm{CD}_{3}, \mathrm{R}=\mathrm{OH} \quad d_{6}$-O-methoxy-pentanedialdoxime-HTyr}

Scheme 3. Derivatization of tyrosol (Tyr) and hydroxytyrosol dialdehydes with unlabelled and labeled methoxyamine.<smiles>[R]c1cc(CC([R])([R])O)ccc1O</smiles>

1. $\mathrm{R}=\mathrm{OH} ; \mathrm{R}^{\prime}=\mathrm{H}$ Hydroxytyrosol

2. $\mathrm{R}=\mathrm{H} ; \mathrm{R}^{\prime}=\mathrm{H}$ Tyrosol

3. $\mathrm{R}=\mathrm{OH} ; \mathrm{R}^{\prime}=\mathrm{D} \quad d_{2}$ - Hydroxytyrosol

4. $\mathrm{R}=\mathrm{H} ; \mathrm{R}^{\prime}=\mathrm{D} \quad d_{2}$ - Tyrosol

Scheme 4. Labelled and unlabelled hydroxytyrosol and tyrosol.

A renowned antioxidant present in olive oil is the secoiridoid oleuropein (OLP). A number of studies have recognized that a diet rich in olive oil, particularly unrefined oils, provides a healthy prevention of artery wall thickening as a consequence of low-density lipoprotein (LDL) oxidation process. This beneficial effect has been associated to the presence of oleic and linoleic monounsaturated fatty acids and to the action of potent antioxidants such as tocopherols and the "polyphenols". ${ }^{99}$ OLP, a secondary metabolite of terpenoid origin, is the main iridoid of the "phenolic pool" 60 of Olea europaea, whose activity is likely associated to its $o$-dihydroxybenzene (catechol) moiety. The same moiety is shared by hydroxytyrosol which is formed by enzymatic degradation of the intact secoiridoid and exhibits similar 
redox activity. Other phenolic compounds, such as tyrosol, caffeic acid, etc., account for the radical scavenger effect of virgin olive oil; ${ }^{61}$ however, attention has been paid to the actual content of oleuropein in foodstuffs due to its therapeutic action. ${ }^{62}$ An ESR study performed on the pure molecule has demonstrated that OLP can likely be exhibited in particular physiological conditions a pro-oxidant activity. ${ }^{63}$

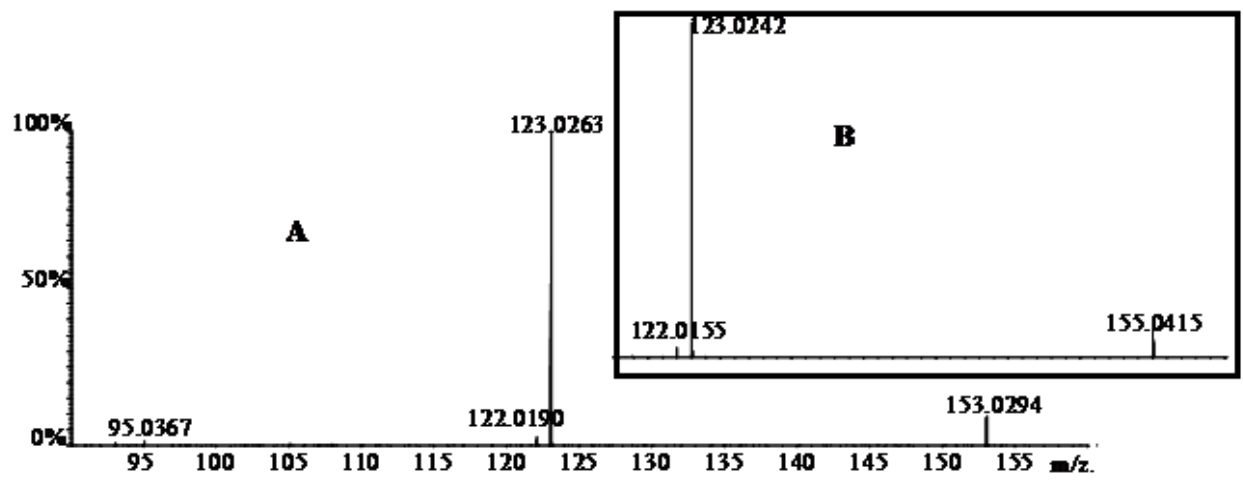

Figure 12. ESI (-) MSMS spectra of compound HTyr (A) and labelled HTyr (B,insert).
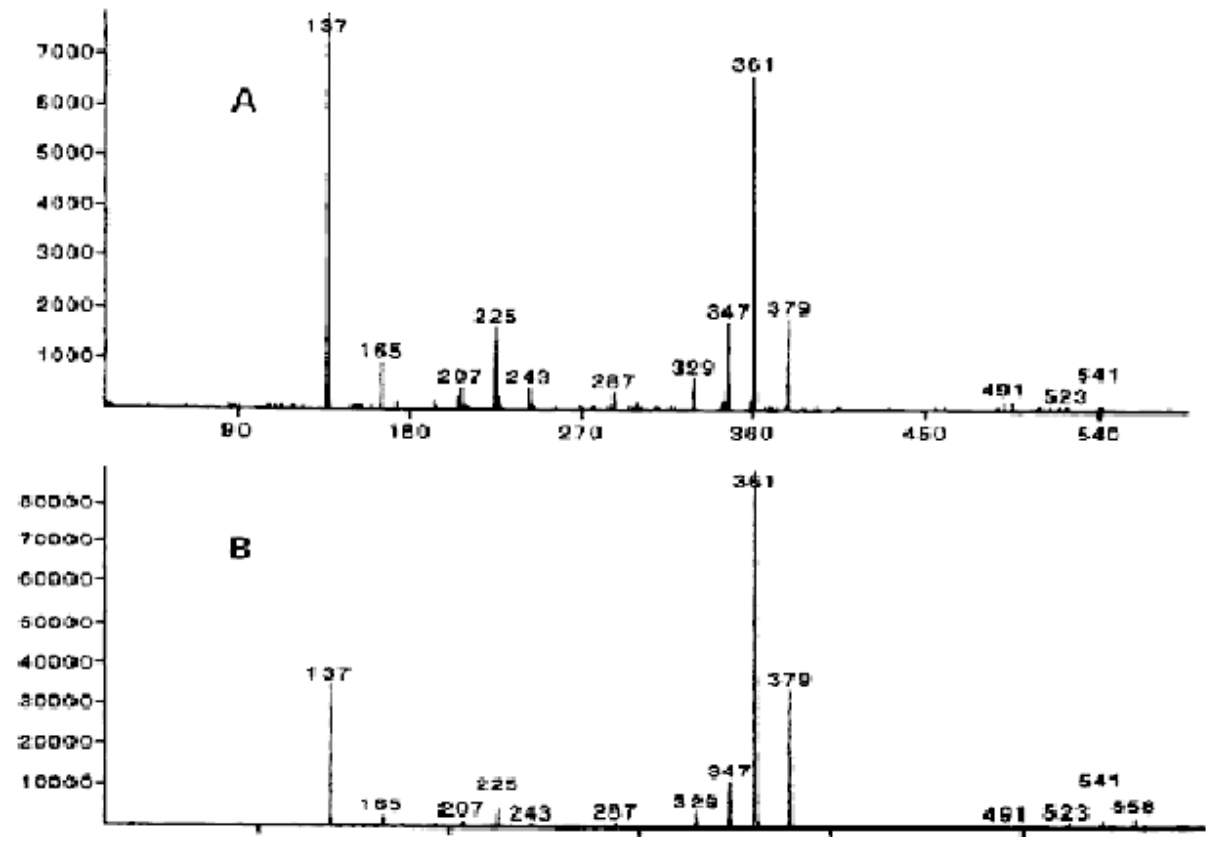

Figure 13. ESI tandem mass spectra of OLP commercial standard. Product ion spectra (A) from $[\mathrm{M}+\mathrm{H}]^{+}$ and (B) from $\left[\mathrm{M}+\mathrm{NH}_{4}\right]^{+}$precursors. 
A comprehensive MS characterization of OLP, showed the advantage of sampling $\left[\mathrm{M}+\mathrm{NH}_{4}\right]^{+}$ ammonium adduct either for the sensitivity of the analytical method and for the peculiar gas-phase chemistry of the species as shown by low and high energy collision experiments. It was shown that the loss of ammonia neutral and the consequent formation of the $[\mathrm{M}+\mathrm{H}]^{+}$ species was controlling the unimolecular dissociations (Figure 13). ${ }^{64}$

The analytical data suggested, therefore, that the $\left[\mathrm{M}+\mathrm{NH}_{4}\right]^{+}$species of oleuropein might correspond to a mixture of different structures and react, in the gas phase, through the elimination of neutral ammonia to give a transient $[\mathrm{M}+\mathrm{H}]^{+}$which possesses enough internal energy to undergo further fragmentation. Oleuropein is extremely reactive in the experimental conditions leading to the preparation of olive oil from drupes, mainly due to the action of glycosidase and esterase enzymes. Therefore its assay in oils derived from different cultivars and different procedures could be considered a marker of process and of good practise. The validated process previous discussed was therefore applied to the monitoring of OLP in oils formed from different cultivars at different ripening stage. (Figure 14). ${ }^{65}$



Figure 14. Ripening-phase-dependent oleuropein content in virgin olive oils of Cassanese and Carolea cultivars. Oleuropein assay in olive oil.

The importance of oleuropein has prompted the development of an absolute method for its assay in olive oil, based on APCI-MS/MS and isotope dilution. ${ }^{66}$

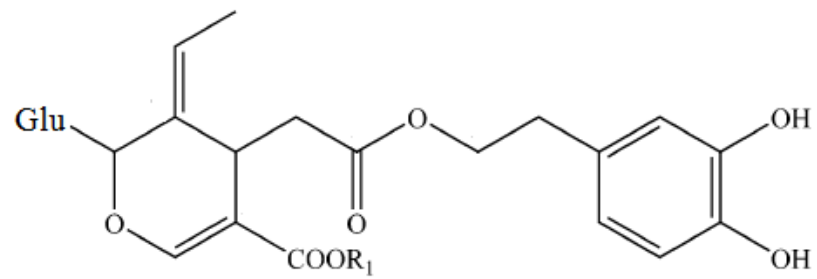

Scheme 5. $\mathrm{R}_{1}=\mathrm{CH}_{3}-\mathrm{OLPd}_{0} ; \mathrm{R}_{1}=\mathrm{CD}_{3}-\mathrm{OLPd}_{3}$ 
The sensitivity and accuracy of the method allowed the discrimination of olive oil samples with very similar content of OLP. In particular filtered and not-filtered products from different southern Italian regions, located at latitude ranging between $\left(38^{\circ} 57^{\prime} \mathrm{N}\right)$ and $\left(42^{\circ}\right.$ $28^{\prime} \mathrm{N}$ ), were discriminated by the amount of OLP which was in the range of $0.093 \div 0.225$ and $0.116 \div 0.344$ ppm, respectively.

A similar approach was exploited in the characterization, at the molecular level, of olive oils produced from the whole fruits or from stoned olives obtained from a pilot plant. ${ }^{67}$ The absolute amount of OLP was $3 \div 4$ times higher in stoned oils, total phenol content showed a similar behaviour, whereas the amount of $\alpha$-tocopherols was higher in oils obtained by conventional milling procedures. The total phenol content should be enhanced either when more lipophilic aglycones are formed, after the removal of the hydrophilic sugar moiety, and, likely, when oleocanthal ${ }^{55}$ and its hydroxytyrosol homologue are obtained after deglycosylation and demethylation at position 11 of oleuropein..$^{68}$ This effect, indirectly, resembles the action of cell-wall-degrading enzymes added to the paste to improve the olive oil quality. ${ }^{69}$



Figure 15. ESI-MS (-) spectra taken directly from the solution containing OLP and the water extracts of Carolea pits after $60 \mathrm{~min}$. of incubation.

The main technological difference in the production of the sampled oils was represented by the presence or absence of stones during the manufacturing procedure. It was therefore checked, always by LC-MS/MS, the action of water extracts of crushed stones on pure samples of OLP was therefore planned. The extracted ion chromatogram showed, after 60 min of incubation, that elenoic acid and its open chain isomers were formed whereas hydroxytyrosol was not detected (Figure 15). This was considered an evidence of the preponderant presence of glucosidases in the stone enzyme pool. The quality of olive oils in relation to the identification and assay of the nutraceutical pool has been recently performed by the new tools of ambient ionization mass spectrometry. A low temperature plasma (LTP) ion source has allowed a rapid screening of free fatty acids, selected bioactive phenolic compounds, volatiles and adulterants in virgin olive oils (Figure 16). $\cdot^{70}$ 

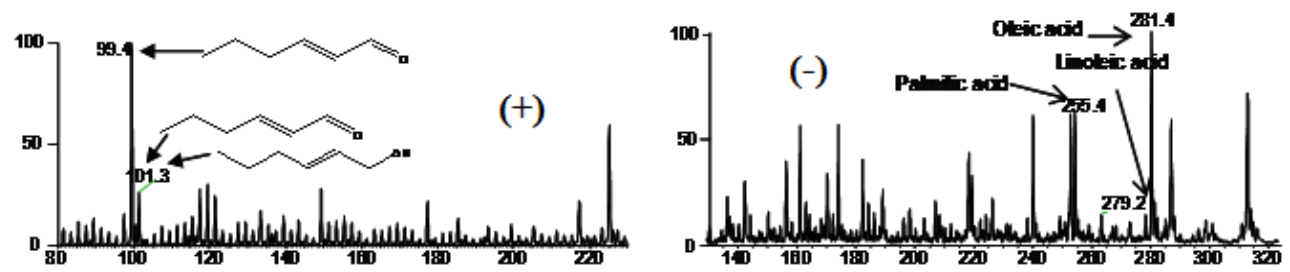

Figure 16. LTP-MS full scan spectrum of crude virgin olive oil in the positive (+) and negative ionization mode (-).

The peculiarity of the method is also represented by the possibility of analyzing each component present in the full scan spectrum with conventional MS/MS devices. Further, another ion source related to the ambient mass spectrometry techniques, named paper spray, has been used for olive oil rapid analysis. The latter, allowed for qualitative determination of several analytes as well as for their assay adding a suitable labeled internal standard. Currently, this technique is applyed also to the is situ derivatization of olive oil (e.g., by methoxyamine), allowing the formation of mass tags and increasing the ionization efficiency for such king of molecules (e.g., oleopentanedialdehydes) in less than one minute.



Figure 17. Variation of the distribution of the aroma markers with the harvesting time in the olive oils produced from two different southern Italy cultivar.

The volatile fraction of olive oil is considered as a source of quality data either for its organoleptic properties and for its correlation to genetic, agronomic and environmental 
factors. Aroma components of products of plant origin are dependent on genetic, agronomic, and environmental factors. ${ }^{71}$ The identification and assay of the terminal species of the "lipoxygenase pathway" which are present in the volatile fraction of olive oils, has been performed by electron impact and/or chemical ionization mass spectrometry in a GC/MS Ion Trap apparatus. The quantitative data for each compound were subjected to principal component analysis to characterize the different cultivars of this work. PCA methodology was applied to confirm the hypothesis that the five selected markers of specific lipoxygenase oxidation could be used to differentiate the various cultivars (Figure 17). ${ }^{72}$ The same method was applied to distinguish the origin of experimental oils produced from drupes harvested in different areas of Italian Calabria region and of Tunisia. An easy discrimination was achieved from each cluster of samples. Olive oils produced from irrigated and nonirrigated farms in Tunisia were also clearly distinguishable. ${ }^{73}$ Quality and safety of oil is also associated to the presence of dangerous organic residues such as phthalates (PAEs). These compounds tend to be distributed mostly in fatty foods and this can cause the presence of remarkable amounts of PAEs in olive oil. Their determination in fatty matrices represents, therefore, a very important goal for the consumers' health and confidence. A rapid method for the analysis of phthalates in olive oil by GC-MS/MS after a GPC clean-up has been developed which exploits the capability of tandem mass spectrometry (GC-MS/MS) for the unequivocal confirmation and accurate quantification of PAEs at low limits of detection (LOD) levels in fatty matrices without the need for a liquidliquid extraction prior to GPC and for SPE clean-up following GPC. ${ }^{74}$ The interest in the identification of secondary metabolites by MS methods has been extended to different olive tissues. An extensive investigation, by means of high-resolution tandem mass spectrometry (MS/MS) has shown that some of these micro components are strongly cultivar dependent, and probably ripening dependent, while others are widespread and present in all the analyzed cultivars. ${ }^{75}$ The new secoiridoid metabolites found in drupes reveal that the key molecules produced by secondary metabolism of terpenes can be conjugated with hydroxytyrosol, a secondary metabolite of phenol biosynthesis, through the formation of differently structured glucosides. The origin of this new species could be related to transport phenomena which can be different among the various tissues of a given plant. Moreover, more stringent evidence of the biogenetic similarity of the members of different oleaceae families is provided by the discovery of metabolites typical of ligustrum and fraxinus in olive tissues. Secondary metabolites of Olea europaea leaves have been selected as markers for the discrimination of cultivars and cultivation zones by multivariate analysis; moreover, the statistical approach has been exploited to correlated the identity and relative amounts of metabolites in leaves with the harvesting period. The mean values of the concentration of each compound, detected by tandem mass spectrometry were inputted into PCA analysis. The bidimensional plot (Figure 18) shows a shifting along PC1 going from March-April to January which indicates the increase of concentration of compounds on the left of plot and the decrease for methoxytyrosol glucoside and 2-methoxyhydroxytyrosol glucoside. Moreover, position of samples of July at highest score values on PC2 means a decrease of concentration of variables verbascoside and hydroxytyrosol glucoside in leaves harvested in this period..$^{76}$ 




Figure 18. Biplot of principal component scores and loadings for leaves of Carolea cv harvested in March, April, July and January (variables are indicated as $\mathrm{m} / \mathrm{z}$ values).

\section{Author details}

Giovanni Sindona and Domenico Taverna

University of Calabria, Dept. Chemistry, Arcavacata di Rende (CS), Italy

\section{References}

[1] Q.Q Wu, K.X Fan, H. Q Ruan, R. Zeng, C. H. Shieh. Chinese Science Bulletin 2009, 54,732737.

[2] E.M. Usai , E. Gualdi , V. Solinas , E. Battistel Bioresour. Technol. 2010, 101 7707-7712.

[3] G. Sindona, A. Caruso, A. Cozza, S. Fiorentini, E. Marini, A. Procopio, S. Zicari, Curr. Med. Chem. 2012, 19, 4006-4013.

[4] A. De Nino, F. Mazzotti, E. Perri, A. Procopio, A. Raffaelli, G. Sindona, J. Mass Spectrom. 2000, 35, 461-467.

[5] Di Donna L, Benabdelkamed H, Mazzotti F, Napoli A, Nardi M, Sindona G, Anal. Chem., 2011, 83, 6, 1990-1995.

[6] Cooks RG et al., Science, 2006, 311, 5767, 1566-70.

[7] Mazzotti F, Di Donna L, Benabdelkamel H, Gabriele B, Napoli A, Sindona, G, J. Mass Spectreom, 2010, 45, 4, 358-63.

[8] J. Charrow, S. I. Goodman, E. R.G. McCabe P. Rinaldo. Genet. Med. 2000, 2, 267-269. 
[9] J.B. Philillips, J. Xu, J.Chromatogr., 1995, 703, 327.

[10] Harynuk, James 2009, June 18.

[11] SciTopics. Retrieved July 25, 2012.

[12] F. Erni and R.W. Frei, J.Chromatogr. 149, 561 (1978).

[13] Schlosser A, Ripkorn R, Bossemeyer D, and Lehmann WD, Anal. Chem., 2001, 73, 170.

[14] Eiceman GA and Stone JA, Anal. Chem., 2004, 76, 391.

[15] Wu C, Siems WF, Asbury GR, and Hill HH, Jr., Anal. Chem., 1998, 70, 4929.

[16] Clemmer DE and Jarrold MF, J . Mass Spectrom., 1997, 32, 577.

[17] Laboda A, J . Am. Soc. Mass Spectrom., 2006, 17, 691.

[18] Hoaglund-Hyzer CS and Clemmer DE, Anal. Chem., 2001, 73, 177.

[19] Ruotolo BT, Gillig KJ, Stone EG, Russell DH, Fuhrer K, Gonin M, and Schultz JA, Int. J. Mass Spectrom., 2002, 219, 253.

[20] Ruotolo BT, McLean JA, Gillig KJ, Stone EG, and Russell DH, J. Am. Soc. Mass Spectrom., 2005, 16, 158; Srebalus Barnes CA and Clemmer DE, Anal. Chem., 2001, 73, 424.

[21] Kellermann M, Muenster, Zomer P, Mol H, 2009, Am Soc Mass Spectrom 20:1464-1476.

[22] Kaufmann A, Butcher P, Maden K, Widmer M, 2010, Anal Chim Acta 673:60-72.

[23] Di Donna, L; Mazzotti, F; Salerno, R; Tagarelli, A; Taverna, D; Sindona, G, Characterization of new phenolic compounds from leaves of Olea europaea L. by highresolution tandem mass spectrometry, RAPID COMMUNICATIONS IN MASS SPECTROMETRY, 21, 22, 3653-3657, 2007.

[24] Nielen MWF, Van Engelen MC, Zuiderent R, Ramaker R, 2007, Anal Chim Acta 586:122-129.

[25] Kaufmann A, The current role of high-resolution mass spectrometry in food analysis, Anal. Bioanal. Chem., 2012, 403:1233-1249

[26] Grimalt S, Sancho J, Pozo OJ, Hernández F, 2010, J Mass Spectrom 45:421-436.

[27] Kaufmann A, Butcher P, Maden K, Widmer M, 2007, Anal Chim Acta 586:13-21.

[28] S. M. Innis, Adv. Nutr. 2011, 2, 275-283.

[29] Amending Regulation (EEC) No 2568/91 on the characteristics of olive oil and oliveresidue oil and on the relevant methods of analysis. COMMISSION REGULATION (EU) No 61/2011 of 24 January 2011.

[30] W. Moreda, M.C. Pérez-Camino and A. Cert.. Grasas y Aceites 2003, 54, 175-179; P. J. Kalo, A. Kemppinen,. Eur. J. Lipid Sci. Technol. 2012, 114, 399-411.

[31] O. Schreiberova, T. Krulikovska, K. Sigler, A. Cejkova and T Rezanka. Lipids 2010, 45,743756 .

[32] L. Mannina, M. Patumi, N. Proietti, D. Bassi, A. L. Segre.. J. Agric. Food Chem. 2001, 49, 2687-2696.

[33] A. Ranalli, L. Pollastri, S. Contento, G. Di Loreto, E. Iannucci, L. Lucera, F. Russi, J. Agric. Food Chem. 2002, 50, 3775;G. Vigli, A. Philippidis, A. Spyros, P. Dais, J. Agric. Food Chem. 2003, 515715.

[34] P. Fronimaki, A. Spyros, S. Christophoridou, P. Dais, J. Agric. Food Chem. 2002, 502207.

[35] K. Nagy, D. Bongiorno, G. Avellone, P. Agozzino, L.Ceraulo, K. Vekey. J.Chromatogr. A, 2005, 1078, 90-97. 
[36] P. B. Chapagain and Z. Wiesman. MALDI-TOF/MS. J. Agric. Food Chem. 2009, 57, 11351142.

[37] G. Ciafardini, B.A. Zullo, A. Iride. Food Microbiology. 2006, 15, 2360.

[38] B.A. Zullo, G. Cioccia, G. Ciafardini. Food Microbiology. 2010, 27,1035.

[39] P. Agozzino, G. Avellone, D. Bongiorno, L. Ceraulo, S. Indelicato, K. Vèkey.. J Mass Spectrom. 2010, 45, 989.

[40] C.M. Kalua, M.S. Allen, D.R. Bedgood Jr, A.G. Bishop, P.D. Prenzler, K. Robards Food Chemistry. 2007, 100, 273.

[41] H. L. Ngo, K. Jones, T.A. Foglia.. JAOCS, 2006, 83, 629.

[42] C. Saiz-Jimenez, B. Hermosin. J Anal Appl Pyrol, 1999, 49, 349 - 357.

[43] D. Tsuchiy, Y. Murakamib, Y. Ogomaa, Y. Kondob, R. Uchio, S. Yamanaka. J. Mol. Catal. B-Enzym. 2005, 35, 52.

[44] M.Attya, A. Russo, E. Perri, and G. Sindona J. Mass Spectrom, 2012, in press.

[45] USDA National Nutrient Database for Standard Reference, Release 15, 2003, www.nal.usda.gov/fnic/foodcomp/Data/SR15/sr15/.html.

[46] Matthaus B, Bruhl L, Comparison of the different methods for the determination of oil content in oilseeds, JAOCS, 78, 95-102, 2001.

[47] Official Methods and Recognized Practices of the American Oil Chemists' Society, $5^{\text {th }}$ ed., Firestone D, Eds., AOCS Press., Champaign, 1998.

[48] Official Methods of Analysis, 17th ed., AOCS International, Gaithersburg, MD, 2000.

[49] Official Methods and Recognized Practices of the American Oil Chemists' Society, $5^{\text {th }}$ ed., Firestone D, Eds., AOCS Press., Champaign, 1998.

[50] Folch J, Lees M, Stanley GHS, A simple method for the isolation and purification of total lipids from animal tissue, J. Biol. Chem., 226, 497-509, 1957.

[51] Ways P, Hanahan DJ, Characterization and quantification of red cell lipids in normal man, J. Lipid Res., 5, 318-328, 1964.

[52] A. De Nino, L. Di Donna, F. Mazzotti, E. Perri, A. Raffaelli, G. Sindona and E. Urso in Biologically-active Phytochemicals in Food Analysis, Metabolism, Bioavailability and Function, Royal Society of Chemistry, Cambridge 2001, 131.

[53] A. Procopio , C. Celia , M. Nardi ,M. Oliverio , D. Paolino , G. Sindona, J Nat Prod. 2011;74, 2377-81.

[54] Servili and Montedoro, 2002, Europ. J. of Lipid Science and Tech., 104, 602-613.

[55] Montedoro, G.; Servili, M.; Baldioli, M.; Selvaggini, R.; Miniati, E.; Macchioni, A. J. Agr. Food Chem. 1993, 41, 2228-2234.

[56] Attya et al, Molecules, 15, 12, 8734-8746, 2010.

[57] Di Donna et al. Anal. Chem., 2011, 83, 1990-1995.

[58] F. Mazzotti, H. Benabdelkamel, L. Di Donna, L. Maiuolo, A. Napoli and G. Sindona. Assay of tyrosol and hydroxytyrosol in olive oil by tandem mass spectrometry and isotope dilution method Food Chem. 2012, 135, 1006-1010.

[59] Halliwell, B.; Aeschbach, R.; Loliger, J.; Aruoma, O. I. Food Chem. Toxicol. 1995, 33, 601.

[60] Servili, M.; Salvaggini, R.; Esposto, S.; Taticchi, A.; Montedoro,G.; Marozzi, G. J. Chromatogr. A 2004, 1054, 113-127.

[61] Roche, M.; Dufour, C.; Mora, N.; Dangles, O. Org. Biomol. Chem. 2005, 3, 423-430. 
[62] Lee-Huang, S.; Zhang, L.; Huang, P. L.; Chang, Y. T.; Huang, P. L. Biochem. Biophys. Res. Commun. 2003, 307, 1029-1037.

[63] A. Mazziotti, F. Mazzotti, M. Pantusa, L. Sportelli and G. Sindona J. Agric. Food Chem. 2006, 54, 7444-7449(15).

[64] A. De Nino, N. Lombardo, E. Perri A. Procopio, A. Raffaelli and G.Sindona. J Mass Spectrom. 1997, 32, 533-541.

[65] E. Perri, A. Raffaelli, and G. Sindona J. Agric. Food Chem. 1999, 47, 4156-4160.

[66] A. De Nino, L. Di Donna, F. Mazzotti; I. Muzzalupo, E. Perri,; A. Tagarelli, Anal. Chem. $2005,77,5961-5964$.

[67] A. De Nino, L. Di Donna, F. Mazzotti, A. Sajjad, G. Sindona, E. Perri, A. Russo, L. De Napoli, L. Filice. Food Chem. 2008, 106, 677-684.

[68] Beauchamp, G. K.; Keast, R.S. J.; Morel, D.; Lin, J.; Pika, J.; Han, Q.; Lee, C. H.; Smith, A. B.; Breslin, P. A. S. Nature, 2005, 437, 45-46.

[69] Vierhuis, E.; Servili, M.; Baldioli, M.; Schols, H.A.; Voragen, A.G.H.; J Agric Food Chem. 2001, 49, 1218-1223.

[70] J. F. Garcia-Reyes, F. Mazzotti. J. D. Harper, N. A. Charipar, S. Oradu, Z. Ouyang, G. Sindona and R. G. Cooks. Rapid Commun. Mass Spectrom. 2009; 23, 3057-3062.

[71] F. Angerosa, N. d'Alessandro, C. Basti, R. Vito, J. Agric. Food Chem. 1998, 46, 2940-2944; F Angerosa; C. Basti, R. Vito, J. Agric. Food Chem. 1999, 47, 836-839.

[72] C. Benincasa, A. De Nino, N. Lombardo, E. Perri, G. Sindona, and A. Tagarelli. J. Agric. Food Chem. 2003, 51, 733-741.

[73] B. Cavaliere, A. De Nino, F. Hayet, A. Lazez, B. Macchione, C. Moncef, E.Perri, G. Sindona, A. Tagarelli. J. Agr. Food Chem. 2007, 55, 1454.

[74] B. Cavaliere, B. Macchione, G. Sindona and A Tagarelli. J. Chromatogr. A, 2008, 1205, 137-143)

[75] L. Di Donna, F. Mazzotti, A. Napoli, R. Salerno, As. Sajjad and G. Sindona Rapid Commun. Mass Spectrom. 2007; 21, 273-27.

[76] L. Di Donna, F. Mazzotti, A. Naccarato, R. Salerno, A. Tagarelli, D. Taverna and G. Sindona. Food Chem. 2010, 121, 492-496. 